\title{
Thyroidectomy in Octogenarians
}

\author{
Ali K. Mahrous' ${ }^{1^{*}}$, Mohamed A. Ellabban², Liam Brennan³ \\ ${ }^{1}$ Department of Otolaryngology, Head and Neck Surgery, Addenbrookes University Teaching Hospital, Cam- \\ bridge, UK. ${ }^{2}$ Department of Surgery, Faculty of Medicine, Suez Canal University, Ismailia, Egypt. ${ }^{3}$ Department of \\ Anaesthesia, Addenbrookes University Teaching Hospital, Cambridge, UK
}

\begin{abstract}
Background: there is a strongly held belief that thyroid surgery on octogenarians is more risky at younger patients. This study aims at investigating whether this assumption is true or not.

Objective: to evaluate the safety of thyroid surgery in the octogenarian population. Materials and Methods: We retrospectively examined our database for those patients who underwent thyroidectomy at the age of 80 years or above between 2004 and 2009. Data were collected regarding patients' age, gender, presenting symptoms, comorbidities, preoperative investigations including Fine Needle Aspiration Cytology (FNAC), whether they underwent total, completion or hemithyroidectomy, postoperative complications, the need for postoperative critical care and histology. Results: 23 patients were identified. All patients had preoperative ultrasound scan of the neck and six had a CT scan of the neck and chest to assess for retrosternal extension. No postoperative complications were reported except for one patient with hematoma and another with right vocal cord palsy. Conclusion: Thyroidectomy in octogenarians is a safe procedure. The incidence of complications in our series is not higher than those in younger population.
\end{abstract}

Keywords: Thyroid, surgery, elderly, goitre

\section{Introduction}

The elderly represents one of the most rapidly growing subsets in the population of Western countries. In the United States alone, there are 35 million people aged more than 65 years. The significant increase in the elderly population has important implications for thyroid surgery. This age group has a higher incidence of neoplasms, and other diseases than younger patients. Furthermore, medical and surgical care for these patients are more complex because of the increased likelihood of comorbidities, which also have a profound influence on the costs of health care ${ }^{(1)}$. Age greater than 70 years is an independent risk factor for complications after general surgery procedures ${ }^{(2)}$. Therefore, indications for surgery in elderly patients should be considered carefully and weighed against the severity of the disease, existing co-morbidities with particular consideration to the postoperative outcome. Increased age is associated with a higher incidence of thyroid diseases ${ }^{(3)}$. The most common indications for thyroid surgery are compressive symptoms, suspicion or confirmation of malignancy, and hyperthyroidism that is resistant to anti-thyroid drugs. However, some surgeons and endocrinologists prefer to delay the surgical procedure 
in the elderly because of the risks of surgery and treat the thyroid disease conservatively with medical or radio-iodine therapy. The incidence of thyroid cancer has increased in recent years in the United States and Europe, with a significant increased incidence after the Chernobyl accident ${ }^{(4,5)}$. It is well established that age plays a fundamental role as a prognostic factor in differentiated thyroid carcinoma ${ }^{(6)}$. Furthermore, some investigators have reported a higher risk of developing cardiac arrhythmias and osteoporosis in patients older than 60 years who present with a subclinical hyper functioning thyroid nod$u_{l}{ }^{(7)}$. For these reasons, any delay in performing surgery for suspicious thyroid pathology could expose the elderly patient to risks related to prolonged medical therapy, subclinical or developed hyperthyroidism and metastasis in the case of malignancy ${ }^{(1)}$. The aim of our study is to examine the safety of thyroid surgery in octogenarians, an area that has not been examined previously.

\section{Subjects and Methods}

We retrospectively examined our database for those patients who underwent thyroidectomy at the age of 80 years or above between 2004 and 2009. Twenty-three patients underwent thyroid surgery in Cambridge University Teaching Hospital. Data were collected regarding patients' age, gender, presenting symptoms, co-morbidities, investigations including preoperative FNAC, whether they underwent total, completion or hemi-thyroidectomy, postoperative complications, the need for postoperative critical care and histology. The inclusion criteria: patients who were 80 year-old or above and who were deemed to withstand surgery. All our surgeries were open with no endoscopic technique in any of our patients. Following the pre- operative anaesthetic assessment, the procedure was performed in the following manner: The patient was put in supine position with "shoulder roll" to reasonably extend the neck. 2) Transverse skin incision along a skin crease of about $4 \mathrm{~cm}$ long. The siting of the incision was midway between suprasternal notch and cricoid. This followed infiltration of the skin with an average of $5 \mathrm{mls}$ of $2 \%$ Lidocaine : $1 \%$ Adrenaline $1 / 800,000$. A size " 10 " blade was used to cut the skin. The subcutaneous tissue was dissected with the use of Monopolar Diathermy with "Colorado" needle going through the platysmal layer. 3) A "subplatysmal" plane was created and the flap was lifted superiorly until thyroid notch is exposed and inferiorly down to the suprasternal notch. 4) The mid-line was identified and strap muscles were divided with the use of monopolar diathermy. This was followed by the peeling the strap muscles off the thyroid capsule. 5) With the use of the harmonic scalpel, the upper pole of the thyroid lobe was divided. Then, the upper pole was retracted inferiorly where the superior parathyroid gland was looked for. 6) The next step was to identify the middle thyroid artery and it was taken as a guide to identify the recurrent laryngeal nerve. Simon's triangle is formed by the common carotid artery laterally, inferior thyroid artery superiorly, and esophagus medially, and the recurrent laryngeal nerve can be seen coursing upward in this triangle to enter the larynx. The nerve usually appears as a white cord with fine red vasa nervosum coursing over its surface. Dissection should proceed directly over the recurrent laryngeal nerve to expose it along its course to the larynx. If the recurrent laryngeal nerve remains difficult to identify, the possibility of a nonrecurrent laryngeal nerve should be considered. 7) Once the nerve was identified, the rest of the ipsilateral lobe was removed with the assistance of harmonic 
scale. 8) The anaesthetist was asked to raise the intrathoracic pressure of the patient to make any occult bleeder visible which then be dealt with by bipolar diathermy. Two or three pieces of surgical were inserted in the pratracheal gutter to aid hemostasis. 9) Cervical plexus block was done routinely at the end of the procedure. 10) The strap muscles were loosely sutured back. The skin was closed in two layers: the platysmal layer with 3/0 undyed vicryl and the skin itself with $4 / 0$ vicryl rapide.

\section{Results}

There were 7 males and 16 females in our series (mean age 84 years; range $81-89$ years.) Sixteen patients presented with a mixture of thyroid related symptoms: (shortness of breath (SOB), feeling of pressure in their neck, dysphagia and tiredness), five patients presented with SOB alone, four with tiredness only, seven with dysphagia, five with pressure symptoms and two were asymptomatic (Fig. 1). Eighteen patients had pre-existing co-morbid conditions (Fig. 2). Ten of these patients had American Society of Anaesthetists grade 2 (ASA 2), seven patients had ASA3 and one patient had ASA4. The main criteria for operative intervention were significant pressure symptoms in thy 1 and thy 2 cases or suspicion of malignancy in thy 3 or above. All patients had a preoperative ultrasound scan of their neck and six had a CT scan of neck and chest, in addition, to assess for retrosternal extension. Out of the 23 patients in our series, five had malignant disease: all were differentiated thyroid cancer (four patients had papillary tumours and one patient had follicularpapillary variant). In terms of the laterality of the surgery, 16 patients had hemithyroidectomy and seven had total thyroidectomy, three of which were completion thyroidectomy. No postoperative complications were reported except one patient had hematoma, which was evacuated, and another with right vocal cord palsy, which had a complete recovery three months after surgery. The majority of patients, who were reported to have Thy 1,2 and 3 on cytology, had benign pathologies on final histology. The exception was that two patients with Thy2 were shown to have papillary thyroid carcinoma and another two patients with Thy3 demonstrated papillary thyroid carcinoma on final histology. The only one Thy 4 patient in our series was reported to have follicular-papillary carcinoma on final histology.

None of the patients required postoperative critical care admission despite the fact that seven of them were classed as $\mathrm{ASA}_{3}$ and one with ASA4. Eight patients had retrosternal extension and 15 displayed no extension. None of the patients with retrosternal extension required a sternal split and the goitre was delivered entirely through the cervical approach. All patients in our cohort were discharged home during the next day of surgery except one patient who developed haematoma, which was evacuated on the second day. The patient was discharged on the third day.

\section{Discussion}

Developed countries are witnessing an increasing life expectancy and with the progressive ageing of the population in the coming decades, it is inevitable that there will be an increasing demand for thyroid surgery in the elderly. Although several studies have demonstrated an increased risk for emergency surgery in patients aged over 60 years ${ }^{(8,9)}$, a recent study showed that thyroid surgery in tertiary centres is safe and efficacious in patients aged over 70 years $^{(1)}$. 


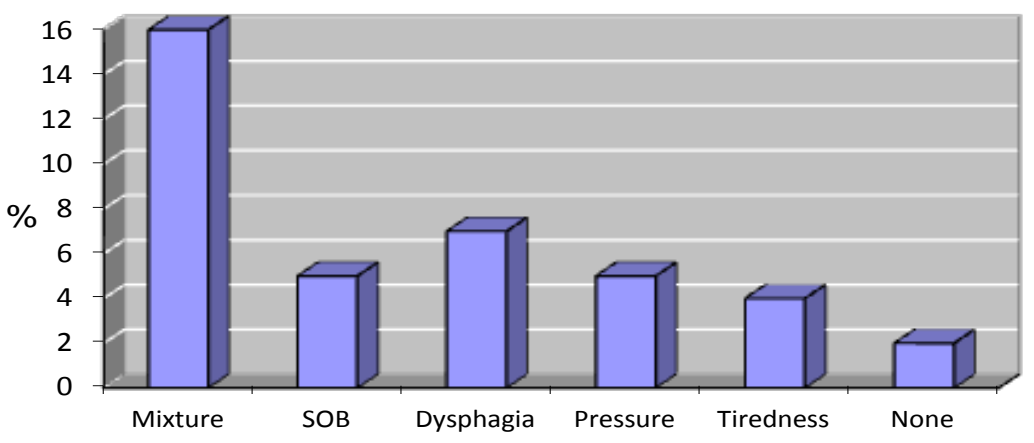

Figure1: The presenting symptoms of the study population

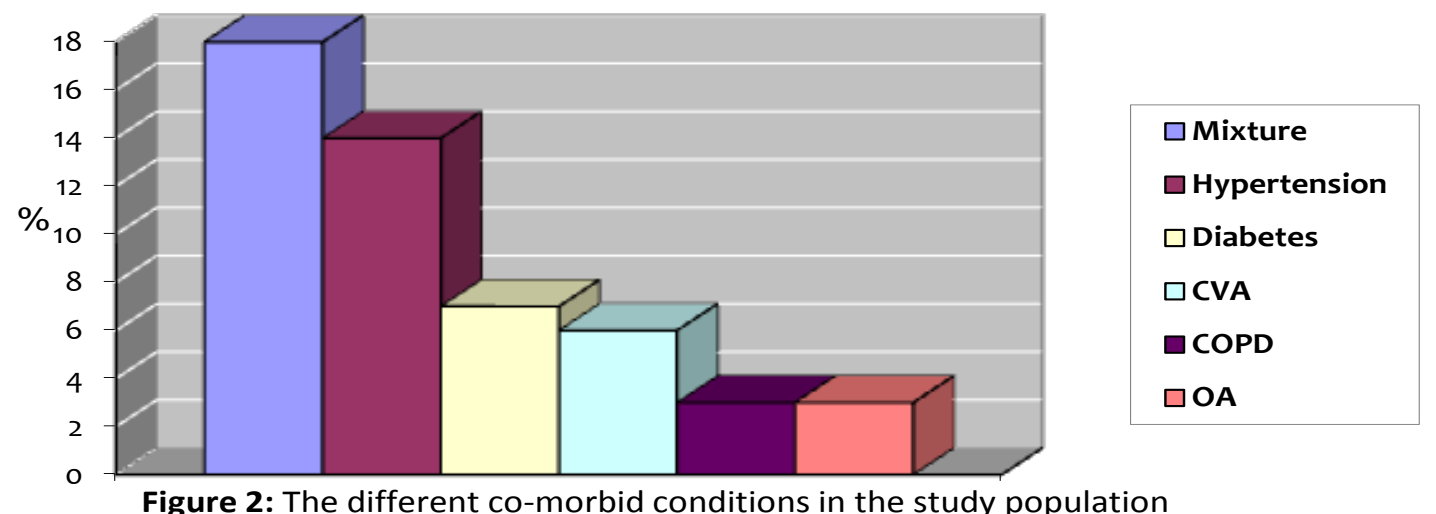

Age plays a major role in the development of malignant thyroid disease and is considered one of the important prognostic factors for outcome ${ }^{(10)}$. Increased aggressiveness of thyroid carcinoma in the elderly is responsible for poorer prognosis of the disease ${ }^{(11)}$. The incidence of thyroid cancer in octogenarians in our small cohort was $21.73 \%$ and all were differentiated carcinomas. This incidence is higher than the reported average in literature, which is around $5 \%^{(12)}$. The improvement in anaesthesia and perioperative care has led to a dramatic decrease in mortality and morbidity ${ }^{(13)}$. We had no mortality or major morbidity in our series. We advocate that this is related to three main factors: patient factors; technical factors and institutional factors. Preoperative optimi- sation of patients' medical conditions is a key factor in minimizing perioperative adverse events. All patients, particularly those with significant co-morbidities, must undergo detailed preoperative assessment by teams led by experienced anaesthetists. This ensures that the individual patient's condition can be optimised and appropriate indications given regarding perioperative risk to aid the consent process. Meticulous and gentle handling of tissues, bloodless surgical field and the use of harmonic scalpel are also contributing factors to a better outcome. Harmonic scalpel has been shown to improve haemostasis as opposed to surgical ligation or bipolar diathermy. This has led to shortening the operating time and minimising blood loss. The latter two 
factors are important to speed up the recovery and to improve the overall outcome of thyroid surgery ${ }^{(14)}$. Other technical factors include small incision $(<4 \mathrm{~cm})$, no strap muscles' division and cervical plexus block all of which improve analge$\mathrm{sia}^{(15)}$. In addition, our institution is a tertiary referral centre and the number of thyroid surgery performed exceeds 180 cases per surgeon per year. This, in turn, is expected to improve the surgical outcome through refining the technique and developing expertise from the multidisciplinary team who manage this group of patients. There are studies showing better outcome in high volume centres, better multidisciplinary and nursing care $^{(16)}$.

It is still debatable whether to undertake total thyroidectomy or hemithyroidectomy in elderly patients with apparent benign disease such as multinodular goitre. This is even more relevant if the patient presents with a dominant nodule on one side of the thyroid. The argument for removing the dominant nodule via a hemi-thyroidectomy would be to alleviate the pressure symptoms and also shorten the operating time which is undoubtedly an important factor in the elderly especially those with significant comorbid conditions ${ }^{(17)}$. In addition, the potential risk for injury to the recurrent laryngeal nerve and hypocalcaemia would be less than with total thyroidectomy. The counterargument is that total thyroidectomy would avoid the risk of disease recurrence and obviate the need for further interventions ${ }^{(18)}$. For patients with retrosternal multinodular goitre and pressure symptom only, we advocate a hemithyroidectomy to "uncork" the thoracic inlet. Given the natural history of recurrence in this age bracket, the chance is low. The major weaknesses of this study are its retrospective nature and the small sample size. Nevertheless, given the relatively advanced age of the patients in our series, one would not expect a huge number of participants. A longer follow up period of analysis is required to determine the impact of recurrence and mortality from concomitant disease in octogenarians. Another limitation of this study is that this group of patients was not compared with the outcomes of a younger cohort. However, the incidence of complications appeared to be similar to that found in younger age groups ${ }^{(19)}$.

\section{Conclusion}

Our results would indicate that thyroidectomy in octogenarians can be performed safely with low levels of morbidity. The operative analysis and postoperative recovery course are comparable to those in younger patients.

\section{Conflicts of Interest}

We all declare that we, the authors, have read and approved the study in question and we declare that there are no potential or actual competing interests.

\section{References}

1. Rafaelli $M$, Bellatone $R$, Princi $P$, De Crea C, Rossi ED, Fadda G, Lombardi CP. Surgical treatment of thyroid disease in elderly patients. Am J of Surg 2010 ; 200 (4):467-472.

2. Goldman L, Caldera DL, Nussbaum SR, Southwick FS, Krogstad D, Murray B, Burke DS, O'Malley TA, Goroll AH, Caplan $\mathrm{CH}$, Nolan J, Carabello B, Slater EE. Multifactorial index of cardiac risk in noncardiac surgical procedures. $\mathrm{N}$ Engl J Med 1977; 297 (16):845- 850.

3. Marchetta FC, Sako K. The enlarged thyroid in the elderly patient. Geriatrics 1968; 23 (5):181 - 188.

4. Jemal A, Siegel R, Ward E, Hao Y, Xu J, Murray T, Thun MJ. Cancer statistics. Ca 
Cancer J Clin 2008;58 (2):71-96.

5. Williams ED. Chernobyl and thyroid cancer. J Surg Oncol 2006; 94 (8): 670- 677.

6. Mazzaferri EL, Kloos RT. Clinical review 128: Current approaches to primary therapy for papillary and follicular thyroid cancer. J Clin Endocrinol Metab 2001; 86 (4):1447-1463.

7. Klein I, Ojamaa K. Thyrotoxicosis and the heart. Endocrinol Metab Cin North Am 1998; 27 (1):51-62.

8. Linn B, Linn M, Wallen N. Evaluation of results of surgical procedure in the elderly. Ann Surg 1982; 195 (1):90- 96.

9. Cook T, Briddon D, Craft T, Jones CB, Horrocks M. An audit of hospital mortality after urgent and emergency surgery in the elderly. Ann R Coll Surg Engl 1997; 79 (5):361-367.

10.Vini L, Hyer SL, Marshall J, A'Hern R, Harmer C. Long term results in elderly patients with differentiated thyroid carcinoma. Cancer 2003; 97 (11):2736- 2742.

11. Girelli ME, Casara D, Rubello D, Mazzarotto R, Saladini G, Busnardo B. Differentiated thyroid cancer (DTC) in the elderly: disease outcome, therapeutic approach and long term results in group of 314 patients. J Endocrinol Invest 1999; 22 (10 suppl):45- 46 .

12. Liénart F, Charret F, Daper C, Dekeyser C, Ducobu J, Malengreau A, Van Coevorden A. [Thyroid nodules: benign or malignant?]. Rev Med Brux. 2011; 32(5):445452.

13. Bliss R, Patel N, Guinea A, Reeve TS, Delbridge L. Age is no contradiction to thyroid surgery. Age Ageing 1999; 28 (4):363-366.

14. Ecker T, Carvalho AL, Choe JH, Walosek $\mathrm{G}$, Preuss KJ. Hemostasis in thyroid surgery: harmonic scalpel versus other techniques--a meta-analysis. Otolaryngol Head Neck Surg. 2010 Jul; 143(1):17-25.

15. Steffen T, Warschkow R, Brändle M, Tarantino I, Clerici T. Randomized controlled trial of bilateral superficial cervical plexus block versus placebo in thyroidsurgery. Br J Surg. 2010; 97(7): 10001006.
16. Pieracci FM,Fahey TJ $3^{\text {rd }}$. Effect of hospital volume of thyroidectomies on outcomes following substernal thyroidectomy. World J Surg. 2008; 32(5):740- 746.

17. Reich DL, Bennett-Guerrero E, Bodian CA, Hossain S, Winfree W, Krol M. Intraoperative tachycardia and hypertension are independently associated with adverse outcome in noncardiac surgery of long duration. Anesth Analg. 2002; 95(2): 273277.

18. Delbridge L, Guinea Al, Reeve TS. Total thyroidectomy for bilateral benign multinodular goitre: effects of changing practice. Arch Surg 1999; 134 (12):1389-1393.

19. Bellantone R, Lombardi $C P$, Bossola $M$, Boscherini M, De Crea C, Alesina P, Traini $E$, Princi $P$, Raffaelli $M$. Total thyroidectomy for management of benign thyroid disease: review of 526 cases. World J Surg 2002; 26 (12):1468 -71. 